\title{
Documentation of Incident Reports by Nurses in Jordanian Accredited Private Hospitals: Types and Causes A Systematic Review
}

\author{
Aiman Gatasheh ${ }^{1}$, Jafar Alkhawaldeh ${ }^{2}$, Dania Bni Hani ${ }^{3}$ \\ ${ }^{I}$ (Critical care nurse, RN,MSC, Manager of quality ,Istiqlal Hospital,Amman,Jordan) \\ ${ }_{2}^{2}$ (critical care nurse, $R N, M S C /$ prospective PhD student at UTS University, Amman, Jordan) \\ ${ }^{3}$ (Nursing Student, the university of Jordan, AMMAN ,Jordan)
}

\begin{abstract}
Incident reports refer to any unplanned events resulting in or with potential for injury, damage or other loss. Many countries have experienced incidents and adverse events that have highlighted failures in their health care systems and various international studies have highlighted the burden of accidents caused by adverse events within the health and social care settings which significantly impacted patients, families and health care system. This review will focus on clarifying the perception of nurses to incidents as a member of the health care workers and their documentation of those incidents by types and causes at Jordanian accredited private hospitals.
\end{abstract}

Keywords: incident reports, incident documentation, incident types and causes.

\section{Introduction}

This chapter will focus on most updated literature's nationally and internationally which focus on identifying incidents by types and causes as well as the difference in perceptions of incidents between health care providers mainly nurses and the importance of Accreditation in improving the culture of patient safety and minimizing incidents .There are too many Incidents, near misses, and sentinel events at health care institutions, but the question is how many of these incidents, near misses and sentinel events are being reported? What actions that have been taken for correction and prevention?

\subsection{Theoretical definition of terms:}

Incident /Adverse event: "An unanticipated, undesirable, or potentially dangerous occurrence in a health care organization".[27]. Sentinel event: "An unanticipated occurrence involving death or major permanent loss of function". [27]. Accreditation: "An evaluation of an organization to assess its compliance with applicable standards and to determine its accreditation status. Which include : Evaluation of documents provided by organization staff that show compliance, verbal information about the implementation of standards or examples of their implementation that enables compliance to be determined, on-site observations by surveyors, tracking of patients through the care process by the tracer methodology, education about standards compliance and performance improvement ".[27 ]Medication error: "Any preventable event that may cause inappropriate medication use or jeopardize patient safety".[27].

\subsection{Operational Definition of terms:}

Incidents: are unintended, unplanned errors that lead or potentially may lead to patient's harm or injury.

Sentinel events: unintended or unplanned errors that may lead to permanent loss of organ function or even death, not related to original disease process.

Accreditation: a process of adopting nationally or internationally standards that meant to enhance the patients care at health care organizations.

Medication error: Any preventable event that may cause inappropriate medication use or interrupt patient safety.

\subsection{Accreditation, documentation and communication:}

Learning from incident reports in the Australian medical imaging setting to determine the type and nature of incidents occurring within medical imaging settings which focused on handover and communication in the radiology events. They found that handover of patient's clinical information to and from medical imaging is full of error, often compromising patient safety and resulting in delayed communication or wrong diagnoses, unnecessary radiation exposure and a waste of limited resources. Problems occurred more frequently during patient preparation $34 \%$, at the time of request for medical imaging $27 \%$ and when communicating a diagnosis $23 \%$. The majority of incidents included in the analysis were reported by radiologists $70 \%$ inpatients accounted for just over half of the patient types 55\% [23]. 
Nursing accreditation system and patient safety study was conducted in Taiwan; results showed that nursing accreditation level is positively related to patient safety indicators. Patient safety is critical to the provision of health services, greater patient safety helps in reducing the lengths of hospital stays, expenses and mortality. The study found that higher nursing accreditation levels may improve patient safety, indicating that nursing accreditation level may be a novel means of improving patient safety.[44].

Study which was conducted in England about which method is more appropriate for hospital staff to detect adverse events and potential adverse events, they found that: Record review detected 26 adverse events (AEs) and 40 potential adverse events (PAEs) occurred during the admission. In contrast, in the same patient group, incident reporting detected 11 PAEs and no AEs. Pharmacy surveillance found 10 medication errors all of which were PAEs. They suggested that one way of reporting adverse events or potentially adverse events is considered not adequate assessment of clinical adverse events and that this method needs to be supplemented with other more systematic forms of data collection, like Structured record review which is carried out by clinicians, provides an important component of an integrated approach to identifying risk in the context of developing a safety and quality improvement program.[18].

One study was conducted to measure hospital progress in reducing error: the impact of external interventions, two or more quality improvement methodologies were being implemented by many of the hospitals, to compare the effectiveness of accreditation in reducing medical errors, the Total Quality Management (TQM) methodology was identified in 80 hospitals, Six Sigma methodology in 26 hospitals and the Baldrige methodology in 14 hospitals. The International Standard ISO 9000 methodology was not identified by any of the hospital administrators. The survey results indicated that (45\%) of the hospital administrators used quality improvement methodologies other than TQM, Six Sigma, or Baldrige. In 22 of these cases, the other methodology was used in conjunction with TQM, Six Sigma, or Baldrige [26].

\subsection{Types and causes:}

One study was conducted at Jordanian hospitals to measure the most frequent types and causes of adverse events, most of the causes were related to, workload and inadequate staffing, technical performance, negligence, poor ethics, poor management, psychosocial job demands, and not clear written guidelines. The major types of adverse events were medication errors which were reported by $56 \%$, then, wrong diagnosis, infections, bedsores and fall. Wrong diagnosis was reported by $21.3 \%$, infections were reported by $21.3 \%$, bedsores were reported by $16 \%$ and falls were reported by $8 \%$ of participants.[24].

In Jordan one study was conducted to describe issues related to medication errors from the nurses' point of view among a sample of 799 nurses. They found that nurses recalled on average 2.2 medication errors per nurse. The main reason for medication errors was related to poor medication labeling and packaging. [35]. According to the American Nurses Association Survey, inadequate staffing was reported by nurses as the top reason for the decline in quality of nursing care. In addition, Oulton, the chief executive officer of the International Council of Nurses states that 'Inadequate staffing leads to tens of thousands of preventable injuries, infections, and deaths each year. According to the Institute of Medicine increased infections, bleeding, cardiac and respiratory failure is associated with inadequate nurse staffing. Furthermore, inadequate staffing frequently leads to a stressful work environment with an increased potential for AEs to occur.

Another Study was conducted on 14 major Athens hospitals to discuss factors impeding nurses from reporting adverse events, the main causes for not reporting incidents were: fear of the press, the licensing board, the difficulty in handling incidents, confidence about bringing up incidents and the complaints by patients[37].

Study on Incident Reporting at a Tertiary Care Hospital In Saudi Arabia reveled that the most common major categories of incident reports were procedural variances $(37 \%)$ followed by behavior and communication incidents $(34 \%)$ and hazardous and safety incidents (10\%). In the ICU, the most common major categories were behavior and communication incidents $(31 \%)$, followed by procedural variances $(21 \%)$, hazardous and safety incidents (13\%), medication errors (13\%), needles and sharps injuries (8\%), equipment incidents (7\%), and intravenous complications $(6 \%)$. There were no reported incidents related to falls or security variances in the ICU.[1]. One of Canadian study was conducted to measure the "Adverse events among medical patients after discharge from hospital revealed that the most common adverse events were therapeutic errors $16 \%$ and nosocomial infections $11 \%$. Out of the 76 patients, 38 had an AE that was either preventable or ameliorable. The AE severity ranged from symptoms only $68 \%$ or symptoms associated with a nonpermanent disability $25 \%$ to permanent disability $3 \%$ or death $3 \%$ [17] .

Another study discussed the general practice errors in United Kingdom (UK): development of an error classification and pilot study of a method for detecting errors, study was conducted on June 2002, results were very optimistic regarding measuring the type of errors in general practice which results in classifying errors in 6 categories, prescriptions, communication, appointments, equipment, clinical care, and 'other'.

Prescription error count $42.2 \%$ and only $6 \%$ of these errors were related medication errors, and $2.6 \%$ clinical errors, and overall rate of errors among general practice was 75.6 /1000 appointment. Five doctors, one 
nurse, one pharmacist, and 11 administrative staff participated and 65 events were recorded in eight of the ten notebooks. Feedback from practice members was generally positive. It was noted that some errors occurred frequently and it was difficult to know how to describe some events accurately. There was agreement that a simple error form listing the most common errors would be a better method of recording errors and would make sure most events were recorded. Staff members were not reporting many incidents because of holidays and a lack of enthusiasm from some of the doctors. Suggestions were made about the wording of the categories and classification and new error recording form was produced [43].

\subsection{Nurses vs. physician reporting incidents:}

Study was conducted to identify adverse events and barriers in reporting adverse events in public hospitals of Shiraz, Iran; study was conducted in 13 hospitals. Cross-sectional, analytical design was conducted. The most relevant factors that influenced the reporting of adverse events in the hospitals were identified. Most of the medicine errors (MEs) in the hospitals were associated with nursing practice $67.3 \%$, Since nursing is integral to patient care, nurses make up the majority of healthcare personnel in most settings, and they spend 8 to 12 hours at patient bedside per shift, fatigue and burnout may have led to adverse events. These results were in line with those of the study by Pham et al. indicating that nurses were responsible for $54 \%$ of the adverse events. Therefore, decreasing nurses' workload, holding training workshops, and preparing safe working environments may decrease nurses' errors. The results showed that $55 \%$ of the errors had occurred in the morning shifts in each of the three groups (physicians, nurses, and services). This might be due to the fact that the personnel's workload is higher and they are forced to do their tasks faster at this time. Individuals and organizational factors were considered as the barriers to reporting the MEs. [30]

Observational study was conducted in Netherlands in 2006 till 2007 to identify nature, causes and consequences on unintended events in surgical units, results were shown 33\% were related to medications while $15.6 \%$ were related to equipments. Most accidents had no physical harm consequences on patients but they result in suboptimal care. Most common causes of incidents were human related, but also some were related to organizational and technical issues. $90 \%$ of incidents were reported by nurses and only $8 \%$ were reported by other medical staff [22].

Study was conducted to measure the Attitudes of doctors and nurses towards incident reporting, they found Nurses reported more habitually than doctors due to a culture which provided directives, protocols and the notion of security, whereas the medical culture was less transparent, favored dealing with incidents and was less reliant on directives. Common barriers to reporting incidents included time constraints, unsatisfactory processes, and deficiencies in knowledge, cultural norms, inadequate feedback, beliefs about risk, and a perceived lack of value in the process [28].

A Study which was published in the Joint Commission Journal on Quality and Patient Safety and was conducted between August 2000 till December 2005 to compare errors reporting between nurses and physicians , the hospital portal was well equipped with reporting system accessible for whoever privileged to access the web site showed that errors which were reported by physicians were very little $(1.1 \%)$ comparing to errors which were reported by nurses $(45.3 \%)$, nurses report errors which caused either no harm or temporary harm to their patients, while physician report errors which caused permanent harm or death [42].

\subsection{Individuals Vs organizational attitude and perception toward incident reports:}

Another Study was conducted to measure the Brazilian registered nurses perceptions and attitudes towards adverse events in nursing care and to describe the perceptions and attitudes of registered nurses (RNs) towards adverse events (AEs) in nursing care. Interviews were conducted with nine Intensive Care Unit RNs. Results showed that: The occurrence of AEs is inherent to the human condition but provokes a feeling of insecurity. The occurrence of AEs indicates the existence of failures in health care systematization .The professional's attitudes towards AEs should be integrated to ethical principles. The priority regarding AEs should be the mitigation of harm to patients. Decisions regarding the communication of AEs were determined by the severity of the error. The various subjective perspectives related to the occurrence of AEs require a health care systematization with a focus on prevention. Ethical behavior is essential for the patient's safety. Implications for nursing management Activities aimed at the prevention of AEs should be integrated jointly with both the professionals and the health care institution. A culture of safety, not punishment, and improvement in the quality of care provided to patients should be priorities [13].

A Study was conducted to measure the feedback from reporting patient safety Incidents at NHS trusts hospitals, showed that there are variations in the feedback systems across many of the NHS hospitals. All NHS trusts disseminate risk awareness through newsletters, meetings and training. Staff need to be encouraged to report incidents and to be acknowledged especially when incident are serious and they are expecting to be informed about the change, Information fed back to frontline staff should include examples of changes resulting from the investigation of their incident reports, mainly when their incidents have direct impact on 
Documentation Of Incident Reports By Nurses In Jordanian Accredited Private Hospitals : Types ..

patient safety Organization should track the impact of feedback and ensure lessons are learnt to improve safety by reporting and investigating incidents. One-third of NHS hospitals didn't get any feedback after they wrote incidents while two-thirds of hospitals get feedback to those who reported adverse incidents to tell them how the issue is being handled[ 49]

Another study was conducted in Netherland to find the perception of health professionals' perceptions of patient safety is related to figures on safety incidents or not? they found that All health professions felt that 'communication breakdowns inside the practice' as well as 'communication breakdowns outside the practice' and 'reporting of patient safety concerns' were a threat to patient safety in their work setting. They found little association between the perceptions of health professionals and the number of safety incidents. The only item with a significant relation to a higher number of safety incidents referred to the Perception of 'communication problems outside the practice' as a threat to patient safety. Which indicate that assessment of professional perception can be complementary to safety incidents? In some measures of patient safety, near-misses are incompletely reported and recorded in hospital records); however, these offer good lessons for improving patient safety [7].Thus, both adverse events and near-misses should be included when assessing patient safety [32].

In health services, nurses may perceive patient safety threats better than health-care organization leaders, indicating that nurses are critical to the assurance of patient safety. Patient safety has been frequently associated with nurse staffing, particularly in high-dependency critical care. Another studies showed that patient outcomes are positively associated with nurse numerical skills, nurse educational level and unit tenure [2]. Evidence also suggests that greater nurse turnover reduces patient safety and increase adverse events [3].

\section{Conclusion}

Some of the studies showed that physicians are reporting incidents more than the nurses while many studies showed that nurses are very close to their patients who are spending most of the time with them, so they are more concerns about safety issues and they report the incidents more. Identifying the most frequent types of incidents and the causes behind them is one of the most important aspects of maintaining patient safety at hospitals. Documentation, Communication and Accreditation are positively associated with improving patient safety which can be measured by decreasing the incidents at hospitals. Many studies were focusing on types of incident and some of them focused on identifying the causes behind these incidents.

In Jordan only one study which focused on measuring both types and causes of incident reports in general, other studies related to medication errors also were identifying the types and causes, but up to the researcher knowledge no studies were conducted in Jordan to measure the nurses perception of incidents as one of the health care workers who are working at accredited private hospitals and their documentation of incidents types and causes.

Since most of the Non Accredited Jordanian private hospitals were not tracking the incident reports and they were not measuring the frequency before having the Accreditation. No data available regarding documentation of incident reports in most of the non accredited Jordanian hospitals.

\section{Acknowledgements}

The authors acknowledge the support from the applied science private university for the financial support granted to this research and everyone assist in this project.

\section{References}

[1]. Arabi, Y., Alamry, A., Al Owais, S.M., Al-Dorzi, H., Noushad, S., Taher, S.(2012). Incident Reporting at a Tertiary Care Hospital in Saudi Arabia. Journal of Patient Safety. 8 (2), 81-7.

[2]. Aiken, C., Kanoya, Y., \& Sato, C. (2007). Factors contributing to medical errors and incidents among hospital nurses: nurses' health, quality of life, and workplace predict medical errors and incidents. Industrial Health, 49, 381-388.

[3]. Bae, G.R., Norton, P.G., Flintoft, V., Blais, R., Brown, A., Cox, J.,... Tamblyn, R.(2010).

[4]. The Canadian Adverse Events Study: The incidence of adverse events among hospital 5.patients in Canada. Canadian Medical Association Journal, 170 (11), 1678-1686.

[5]. Barach, P. and Small, S. (2000) "Reporting and preventing medical mishaps: lessons from non-medical near miss reporting", British Medical Journal, vol.320, pp.579-63.

[6]. Bartlett, G., Blais, R., Tamblyn, R., Clermont, R., MacGibbon, B. (2008). Impact of Patient communication problems on the risk of preventable adverse events in acute care settings. Canadian Medical Association Journal. 178, 1555-1562.

[7]. Beckmann, U., Gillies, D.M., Berenholtz, S.M., et al. (2004). Incidents relating to the intra-hospital transfer of critically ill patients. An analysis of the reports submitted to the Australian Incident Monitoring Study in Intensive Care. Intensive Care Medicine. 30 (8), $1579-1585$.

[8]. Christiaans-Dingelhoff, I., Smits, M., Zwaan, L., Lubberding, S., van der Wal, G., Wagner, C. (2011). To what extent are adverse events found in patient records reported by patients and healthcare professionals via complaints, claims and incident reports?. BMC Health Services Research. 11 (49), 1-10.

[9]. Croskerry, P., Sinclair, D. (2001). Emergency medicine: A practice prone to error?. Canadian Journal of Emergency Medicine. 3(4), 271.

[10]. Cross, S., \& Miller, Z. (2007). NHS Scotland Incident Reporting Culture: Extended Study National Summary Report. Glasgow: NHS Quality Improvement Scotland, 1-21.

[11]. Cross, S., \& Miller, Z. (2007). NHS Scotland Incident Reporting Culture: Board Report. NHS Argyll and Clyde, 1-34.

DOI: 10.9790/1959-060207101106 www.iosrjournals.org $\quad$ 104|Page


Documentation Of Incident Reports By Nurses In Jordanian Accredited Private Hospitals : Types ..

[12]. Davis, P., Lay-Yee, R., Briant, R., Ali, W., Scott, A., \& Schug, S. (2002). Adverse events in New Zealand public hospitals I: occurrence and impact. The New Zealand Medical Journal, 115 (1167), 203-209.

[13]. De Freitas, G.F., Hoga, L.A., Fernandes, M. F., González, J.S., Ruiz, M.C., Bonini, B.B.(2011). Brazilian registered nurses perceptions and attitudes towards adverse events in nursing care: a phenomenological study. Journal of Nursing Management. 19, 331-338.

[14]. De Vries, E.N., Ramrattan, M.A., Smorenburg, S.M., Gouma, D.J., \& Boermeester, M.A. (2008). The incidence and nature of inhospital adverse events: a systematic review. Quality \& Safety in Health Care, 17 (3), 216-23.

[15]. Donchin, Y., Gopher, D., Olin, M., Badihi, Y., Biesky, M., Sprung, C.,... Cotev, S. (1995). A look into the nature and causes of human errors in the intensive care unit. Critical care medicine. 23, 294- 300.

[16]. Forster, A.J., Clark, H.D., Menard, A., Dupuis, N., Chernish, R., Chandok, N., ... van Walraven, C. (2004). Adverse events among medical patients after discharge from hospital. Canadian Medical Association Journal, 170 (3), 345-349.

[17]. Forster, A.J., Murff, H.J., Peterson, J.F., Gandhi, T.K., Bates, D.W. (2004). The Incidence and Severity of Adverse Events Affecting Patients after Discharge from the Hospital. Annals of Internal Medicine. 138 (3), 161-7.

[18]. Friedman, S. M., Provan, D., Moore, S., \& Hanneman, K. (2008). Errors, near misses and adverse events in the emergency department: what can patients tell us? Canadian Journal of Emergency Medicine, 10, 421-427.

[19]. Fukuda, H., Imanaka, Y., Hirose, M., et al. (2010). Impact of system-level activities and reporting design on the number of incident reports for patient safety. Quality and Safety in Health Care. 19, 122-127.

[20]. Gawande A, Zinner M, Studdert D, Brennan T.(2003). Analysis of errors reported by surgeons at three teaching hospitals.Surgery; 133: 614-621.

[21]. Gazarian, P. (2008). Nurse Decision Making and The Prevention of Adverse Events. Doctoral Thesis, School of NursingUniversity of Massachusetts.

[22]. Greenberg, C.C., Regenbogen, S.E., Studdert, D.M., et al. (2007). Patterns of communication breakdowns resulting in injury to surgical patients. Journal of the American College of Surgeons. 204, 533-540.

[23]. Hannaford, N., Mandel, C., Crock, C., Buckley, K., Magrabi, F., Ong, M. ,...Schultz, T. (2013). Learning from incident reports in the Australian medical imaging setting: handover and communication errors. The British Journal of Radiology, 86, 20120336.

[24]. Hayajneh, Y.A., Abu AlRub, R.F., \& Almakhzoomy, I.K. (2010). Adverse events in Jordanian hospitals: types and causes. International Journal of Nursing Practice, 16, 374-380.

[25]. Helmreich, R. and Merritt, A. (2001). Culture at work in aviation and medicine, Aldershot: Ashgate.

[26]. Hensel, D., Anderson, J., \& Ramanujam, R. (2005). Examining the Importance of Organizational Structure on Voluntary Medical Error Reporting Over Time.

[27]. American Sociological Association Annual Meeting, Philadelphia, 1-18. Hosford, S.B. (2006). Hospital Progress in Reducing Error: The Impact of External Interventions. Hospital Topics, Winter, 86 (1), 9-19.

[28]. Joint Commission International. (2013). http://www.jointcommissioninternational.org. Khammarnia, M. (2014). Adverse events and barriers to reporting in hospitals in Southern Iran. Australian Medical Journal. 7 (11), 445-447.

[29]. Kingston, M.J., Evans, S.M., Smith, B.J., \& Berry, J.G. (2002). Attitudes of doctors and nurses towards incident reporting: a qualitative analysis. Medical Journal of Australia, 181 (1), 36-39.

[30]. Kohn, L.T., Corrigan, J.M., Donaldson, M.S. (2000). To Err is Human: Building a Safer Health System. Institute of Medicine Committee on Quality of Health Care in America- National Academy Press.

[31]. Landrigan, C.P., Parry, G.J., Bones, C.B., Hackbarth, A.D., Goldmann, D.A., \& Sharek, P.J. (2010). Temporal trends in rates of patient harm resulting from medical care. The New England Journal of Medicine, 363, 2124-2134.

[32]. Lawton, R. J., \& Parker, D. (2002). Barriers to incident reporting in a healthcare system. Quality and Safety in Health Care, 11 (1), $15-18$.

[33]. Martijn, L., Harmsen, M., Gaal, S., Mettes, D., van Dulmen, S., Wensing, M. (2013). Are health professionals' perceptions of patient safety related to figures on safety incidents? Journal of Evaluation in Clinical Practice. 19(5):944-7.

[34]. Morimoto, T., Gandhi, T.K., Seger, A.C., Hsieh, T.C., Bates, D.W. (2004). Adverse drug events and medication errors: detection and classification methods. Quality and Safety in Health Care. 13 (4), 306-14.

[35]. Moumtzoglou, A. (2010). Factors impeding nurses from reporting adverse events. Journal of Nursing Management, 18, 542-547.

[36]. Mrayyan, M. T. (2006). Reported incidence, causes, and reporting of medication errors in teaching hospitals in Jordan: A comparative study. Contemporary Nurse. A Journal for the Australian Nursing Profession, 41 (2), 216-232.

[37]. Mrayyan, M., Shishani, K., \& Al-Faouri, I. (2007). Rate, causes and reporting of medication errors in Jordan: nurses' perspectives. Journal of nursing management. 15 (6), 659-670.Olsen, S., Neale, G., Schwab, K., Psaila, B., Patel, T., Chapman, E.J., \& Vincent, C. (2007). Hospital staff should use more than one method to detect adverse events and potential adverse events: incident reporting, pharmacist surveillance and local real-time record review may all have a place. Quality and Safety in Health Care, 16 (1), 40-4.

[38]. Oumt, B. (2010). Leadership in the NHS, Error making and nursing. Nursing Management, 8 (10), 24-27. Oulton, J. A. (2006). Safe Staffing-A Shared Responsibility. International Council of Nurses. 53(1), 11-15.

[39]. Petersen, L., Brennan, T., O'Neil, A. (1994). Does house staff discontinuity of care increase the risk of preventable adverse events?. Annals of Internal Medicine. 121, 866-872.

[40]. Reason, J. and Hobbs, A. (2003). Managing Maintenance Error, Aldershot: Ashgate.

[41]. Regenstein, M. (2004). Understanding the first Institute of Medicine report and its impact on patient safety. In B. J. Youngberg \& M. Hatlie (Eds.), The patient safety handbook. Sudbury, MA: Jones and Bartlett.

[42]. Rogers A.E., Hwang, W.T., Scott, L.D., Aiken, L.H., Dinges, D.F. (2004). The working hours of hospital staff nurses and patient safety. Health Affairs, 23 (4), 202-212.

[43]. Rowin, E. J., Lucier, D., Pauker, S. G., Kumar, S., Chen, J., \& Salem ,D. N. (2005). Does Error and Adverse Event Reporting by Physicians and Nurses Differ?. The Joint Commission Journal on Quality and Patient Safety, 34 (9), 537-545.

[44]. Rubin, G., George, A., Chinn, D.J., Richardson, C. (2002). Errors in general practice: Development of an error classification and pilot study of a method for detecting errors. Quality and Safety in Health Care, 12, 443-447.

[45]. Teng, C., Shyu, Y.-I. L., Dai, Y.-T., Wong, M.-K., Chu, T.-L., \& Chou, T.-A. (2012). Nursing accreditation system and patient safety. Journal of Nursing Management, 20, 311- 318

[46]. Tran, D.T., \& Johnson, M. (2010). Classifying nursing errors in clinical management within an Australian hospital. International Nursing Review, 57, 454-462.

[47]. Van Wagtendonk, I., Smits, M., Merten, H., Heetveld, M.J., \& Wagner, C. (2010). Nature, Causes and consequences of unintended events in surgical units. British Journal of Surgery, 97 (11), 1730-40.

[48]. Vincent, C., Neale, G., \& Woloshynowych, M. (2001). Adverse events in British hospitals: preliminary retrospective record review. British Medical Journal, 322, 517-519. 
[49]. Vincent, C.A., \& Coulter, A. (2002). Patient safety: What about the patient?. Quality \& Safety in Health Care. 11(1), 76-80.

[50]. Wallace, L. (2010). Feedback from reporting patient safety incidents - are NHS trusts Learning lessons. Journal of Health Services Research \& Policy. (15), 75-78.

[51]. Wallace, L.M., Spurgeon, P., Benn, J., Koutantji, M., \& Vincent, C. (2008). Improving Patient-safety incident-reporting systems by focusing upon feedback- lessons from English and Welsh trusts. Health Services Management Research, 22 (3), 129-135.

[52]. Waring, J.J. (2004). Beyond blame: cultural barriers to medical incident reporting. Social Science and Medicine, 60 (9), 1927-35

[53]. Weissman, J., Rothschild, J., Bendavid, E. et al. (2007). Hospital workload and adverse Events. Medical Care. 45, 448-455.

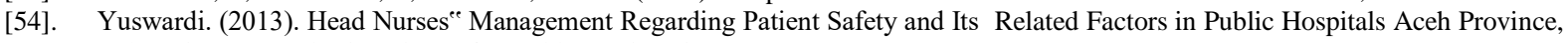
Indonesia. Master thesis, prince of Songklan University. 